\title{
Investigation of the mechanical properties of thin films by nanoindentation, considering the effects of thickness and different coating-substrate combinations
}

\author{
Shaohua Chen*, Lei Liu, Tzuchiang Wang \\ LNM, Institute of Mechanics, Chinese Academy of Sciences, Beijing 100080, China
}

Received 22 September 2003; accepted in revised form 17 March 2004

Available online 2 June 2004

\begin{abstract}
In order to further investigate nanoindentation data of film-substrate systems and to learn more about the mechanical properties of nanometer film-substrate systems, two kinds of films on different substrate systems have been tested with a systematic variation in film thickness and substrate characteristics. The two kinds of films are aluminum and tungsten, which have been sputtered on to glass and silicon substrates, respectively. Indentation experiments were performed with a Nano Indent XP II with indenter displacements typically about two times the nominal film thicknesses. The resulting data are analyzed in terms of load-displacement curves and various comparative parameters, such as hardness, Young's modulus, unloading stiffness and elastic recovery. Hardness and Young's modulus are investigated when the substrate effects are considered. The results show how the composite hardness and Young's modulus are different for different substrates, different films and different film thicknesses. An assumption of constant Young's modulus is used for the film-substrate system, in which the film and substrate have similar Young's moduli. Composite hardness obtained by the Joslin and Oliver method is compared with the directly measured hardness obtained by the Oliver and Pharr method.
\end{abstract}

(C) 2004 Elsevier B.V. All rights reserved.

Keywords: Nanoindentation; Mechanical property; Coating and substrate

\section{Introduction}

Elastic modulus and hardness are two important material parameters. Industry has long relied on indentation techniques for measuring the hardness of a wide range of materials. Recently, depth-sensing indentation techniques are used to study the mechanical properties of thin film on substrate $[1-4]$. Indentation techniques have been of particular interest to thin films used in the semiconductor and magnetic storage industry. As film thicknesses used in these industries continue to decrease, however, it becomes more and more difficult to obtain useful information from these techniques. Due to equipment limitations such as machine resolution, signal-to-noise ratio, tip rounding effects, it is very difficult to obtain meaningful experimental results for indentation depths less than $10 \mathrm{~nm}[2,5]$. In order to obtain

\footnotetext{
* Corresponding author. Tel.: +86-10-62545533-3023; fax: +86-1062561284

E-mail address: chenshaohua72@hotmail.com (S. Chen).
}

intrinsic film properties from larger indentations, one needs to understand how the mechanical properties of the substrate affect measurements of film stiffness and hardness.

Numerous investigators have used both experimental [514] and theoretical methods [15-22] to study how to extract true film properties from nanoindentation of film-substrate systems.

There are several methods for determination of elastic modulus of thin films, especially that based on bending of microbeams and nanoindentation, see Refs. [23-29]. The latter method is advantageous in that it has no special requirements regarding the specimen shape and preparation. However, the indenter penetrating into the material deforms not only the film but usually also the substrate. It is thus the composite modulus that is obtained in such a test and the film modulus must be determined by a suitable processing of the data.

Also, several theoretical methods $[6,16,30-38]$ have been developed to describe the hardness of film-substrate systems. These models are based on the idea that the composite hardness is determined by the weighted average 
of film and substrate hardnesses in proportion to the relative deformed areas or volumes.

In this paper, we consider the nanometer film-substrate systems, including both soft films on hard substrates and hard films on soft substrates. Similar problems have been investigated by Ref. [14], but in this study different thicknesses of films are used, with all the film thicknesses being in the order of sub-micrometers in order to find a true result and to verify the results given by Ref. [14]. Specifically, we study the nanoindentation properties of aluminum and tungsten films on silicon and glass substrates. In the filmsubstrate systems aluminum on glass is included, as is nearly elastically homogeneous. For this kind of filmsubstrate system, a measurement of the contact stiffness during indentation, together with knowledge of the elastic modulus of the system, permits the determination of the true contact area and true hardness, irrespective of the effects of pile-up or sink-in around the indenter but beyond the depth at which the tip round influence is obvious. Knowledge of the true hardness of the film can be used to assess the effects of the elastic modulus mismatch on the nanoindentation properties, by using the measured contact stiffness as a function of depth of indentation relative to the film thickness for films on different substrates. The effects the substrate may have on the determination of mechanical properties of thin films could be investigated through the nanoindentation experiments on the film-substrate systems.

\section{Experiment details}

\subsection{Sample preparations}

Research S-Gun II Turbosystem (Sputtered Film) is used and both $\mathrm{Al}$ and $\mathrm{W}$ are deposited onto the different substrates by sputtering. The base pressure in the chamber prior to sputtering is $5 \times 10^{-7}$ Torr and the sputtering pressure is 1.0 Pa. Three kinds of Al films are deposited on glass and silicon substrates, respectively, and the deposition rates are 110,160 and $160 \AA / \mathrm{min}$, respectively and all under $1000 \mathrm{~W}$ of power. Two kinds of $\mathrm{W}$ films are deposited on glass and silicon substrates also, for which both the deposition rates are $60 \AA / \mathrm{min}$ and both under $500 \mathrm{~W}$ of power.

The three levels of nominal thicknesses of $\mathrm{Al}$ films are 52.3, 244.7 and $850.9 \mathrm{~nm}$, while the $\mathrm{W}$ films have nominal thicknesses of 66.5 and $280 \mathrm{~nm}$. Finally, we obtain 10 samples, that is, $\mathrm{Al}-$ glass and $\mathrm{Al}-\mathrm{Si}$ with three levels of film thickness, 52.3, 244.7 and $850.9 \mathrm{~nm}$, respectively; and $\mathrm{W} /$ glass and W/Si with two levels of film thickness, 66.5 and $280 \mathrm{~nm}$, respectively.

\subsection{Theoretical background of indentation experiments}

The mechanical properties of the substrates and films are characterized using Nanoindentation XP II with a Berkovich indenter tip. Continuous stiffness mode is used in all experi- ments. The indentations are made with a constant nominal strain rate $0.05 \mathrm{~s}^{-1}$. Five indentations are made in each sample and the results presented are the average of these five indentations. Hardness and Young's modulus are first determined by means of the Oliver and Pharr analysis method. It should be noted that Oliver and Pharr's method is for monolithic materials and only for sink-in. However, this kind of method has been the standard method of analysis for nanoindentation and is frequently used for thin film studies. In order to avoid the calculation of contact area, which sometimes causes some errors in the results due to sink-in or pile-up, we will use the method given by Joslin and Oliver [9] and the constant Young's modulus assumption to analyze the experiment data.

With depth-sensing nanoindentation devices, elastic modulus is determined [39] from

$E_{\mathrm{r}}=\frac{\sqrt{\pi}}{2 \beta} S \frac{1}{\sqrt{A}}=\frac{\sqrt{\pi}}{2 \beta} \frac{\mathrm{d} P}{\mathrm{~d} h} \frac{1}{\sqrt{A}}$

$S=\frac{2 \beta}{\sqrt{\pi}} \sqrt{A} E_{\mathrm{r}}$

where $A$ is the projected area of the contact, $\beta$ is a constant that depends on the geometry of the indenter. $S=\mathrm{d} P / \mathrm{d} h$ is the slope of the load-displacement curve at the beginning of the unloading stage and $E_{\mathrm{r}}$ is the reduced Young's modulus, which can be obtained as [18]

$\frac{1}{E_{\mathrm{r}}}=\frac{1-v_{\mathrm{i}}^{2}}{E_{\mathrm{i}}}+\frac{1-v_{\mathrm{f}}^{2}}{E_{\mathrm{f}}}\left(1-e^{-\alpha \frac{t}{a}}\right)+\frac{1-v_{\mathrm{s}}^{2}}{E_{\mathrm{s}}} e^{-\alpha_{a}^{\frac{t}{a}}}$

where $E_{\mathrm{i}}, E_{\mathrm{f}}, E_{\mathrm{s}}$ are Young's modulus of the indenter, film and substrate, respectively. $v_{\mathrm{i}}, v_{\mathrm{f}}$ and $v_{\mathrm{s}}$ are Poisson's ratio of the indenter, film and substrate, respectively. $\alpha$ is a constant related with the indenter geometry. $t$ is the film thickness under the indenter and $a=\sqrt{A}$. It means if the film modulus $E_{\mathrm{f}}$ differs from that of the substrate, $E_{\mathrm{s}}$, then the measured $E_{\mathrm{r}}$ value changes with depth of penetration $h$. If the film has a similar Young's modulus with the substrate, i.e., $E_{\mathrm{f}} \approx E_{\mathrm{s}}$ and $v_{\mathrm{f}} \approx v_{\mathrm{s}}$, then the reduced Young's modulus can be expressed as

$\frac{1}{E_{\mathrm{r}}}=\frac{1-v_{\mathrm{i}}^{2}}{E_{\mathrm{i}}}+\frac{1-v_{\mathrm{f}}^{2}}{E_{\mathrm{f}}}$

Hardness is usually defined as

$H=\frac{P}{A}$

According to Ref. [9] and eliminating the contact area from Eqs. (1) and (5), we get the composite hardness for film-substrate system,

$H=\frac{4 \beta^{2}}{\pi} \frac{P}{S^{2}} E_{\mathrm{r}}^{2}$

From the above equation, one can see that the hardness, $H$, is directly proportional to the parameter $P / S^{2}$ and 
proportional to the square of the reduced modulus, $E_{\mathrm{r}}^{2}$. If the film has a similar Young's modulus with that of the substrate, the reduced modulus, $E_{\mathrm{r}}$ will approximately be a constant, which is called the constant Young's modulus assumption. Using Eq. (6), we could avoid the calculation of contact area and obtain the true composite hardness through the parameter, $P / S^{2}$.

Sometimes, Eq. (6) can be rewritten as

$\frac{P}{S^{2}}=\frac{\pi}{4 \beta^{2}} \frac{H}{E_{\mathrm{r}}^{2}}$

\section{Roughness of the sample surface}

In this study, we choose five samples with $\mathrm{Al}$ and $\mathrm{W}$ films on Si substrates and use AFM to check the roughness of the sample surface. During the test, we choose an area of $5 \times 5 \mu \mathrm{m}$ and adopt the noncontact method, i.e., the tip point of AFM does not contact with the sample surface.

As for the roughness of the film surface of $\mathrm{Al}-\mathrm{Si}$ sample with film thickness of $52.3 \mathrm{~nm}$, the surface is much smooth as the peak value is $6.71 \mathrm{~nm}$ and the valley value is -7.287 $\mathrm{nm}$. Each grain size does not change too much and distributes homogeneously. For the roughness of the Al film surface on Si substrate with film thickness of $244.7 \mathrm{~nm}$, there are some large grains formed and distributed on the film surface. It may be caused by crystallization due to high temperature and relative long sputtering time. The surface is very smooth that the peak value and the valley value are similar to those in $52.3 \mathrm{~nm}$ case except at the site of the unexpected large crystals. For the roughness of $\mathrm{Al}$ film on $\mathrm{Si}$ substrate with film thickness of $850.9 \mathrm{~nm}$, the grain is very clear and much larger than those in the above two samples. Also, large crystals can be found. The surface roughness is smooth relative to the film thickness and the difference between the peak value and the valley value is about $30 \mathrm{~nm}$ except at the site of large crystals.

As for the roughness of W film on Si substrate with film thickness of $66.5 \mathrm{~nm}$, the film surface is very smooth and the difference between the peak and the valley values is about $5 \mathrm{~nm}$ except at the site of the large crystals, which may be formed by crystallization due to high temperature. For the roughness of $\mathrm{W}$ film on $\mathrm{Si}$ substrate with film thickness of $280 \mathrm{~nm}$, the difference between the peak and the valley values is about $20 \mathrm{~nm}$ except at the site of the large crystals.

One should note that the grain size in films becomes larger when the film thickness increases, which would cause Hall-Petch effect on the flow stress [40]. On the other hand, crystallization will increase when the film thickness increases.

Since the crystallization does occur during the sputtering process, we will avoid indentation on the large crystals in order to obtain the true mechanical characteristics of the film-substrate system.

\section{Experiment results}

The Poisson's ratios of the films and substrates in all the calculations on the experiment data are taken as 0.3 since Ref. [41] has shown that the Poisson's ratio has a minor effect on the indentation results. We also analyze the error that is introduced by different Poisson's ratios. According to Eq. (3), if we take the same Young's modulus of the film as that of the substrate, such as Al-glass, and the maximum value of $e^{-\alpha(t / a)}$ in Eq. (3) as the unit, the maximum error of the reduced Young's modulus introduced is about $5 \%$ for $v_{\text {glass }}=0.3$ and $v_{\text {glass }}=0.2$.

In the present study, we first indent only the substrates and obtain the substrates' Young's modulus, hardness and the parameter values, $P / S^{2}$. The maximum indentation depths on the substrates are $2 \mu \mathrm{m}$. Then the film-substrate systems are indented and the indentation depths are twice of the thicknesses of the films.

\subsection{Substrate characterization}

The substrates used in the present study are glass and silicon. Silicon used in the present study belongs to AMEX and $N$ type (100). Its thickness is $525 \pm 25 \mu \mathrm{m}$. Glass is HOYA-SDII glass of thickness $1000 \mu \mathrm{m}$.

As mentioned above, five indentations are made on each sample. The loading and unloading curves for all five indentations on a single substrate repeat very well. When the maximum loading is reached, it will be kept for several seconds to reduce the creep effects. At the same indentation depth, the load on silicon is larger than that on glass, which means that silicon is harder than glass. The hardness and Young's modulus of the bare substrates are calculated from nanoindentation data using the Oliver-Pharr method as shown in Fig. 1. From Fig. 1, the hardness of the silicon is $12.5 \mathrm{GPa}$ and the Young's modulus is $185 \mathrm{GPa}$. The hardness of glass is $8.2 \mathrm{GPa}$ and the Young's modulus is 88.5 GPa. During the indentation, all the curves of hardness and Young's modulus versus the indentation depth are

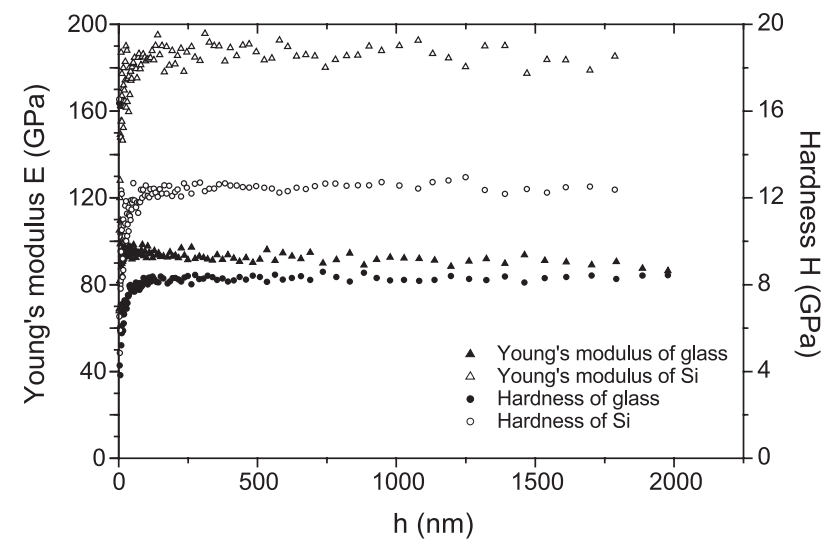

Fig. 1. Hardness and Young's modulus of silicon and aluminum bulk materials 
approximately horizontal lines except at the initial stage, which is influenced by a number of effects, such as the tip rounding, the indenter drift, the determination of zero point, the surface roughness and machine resolution. Generally, the tip rounding is the most obvious one. Usually a Berkovich tip round is about $50 \mathrm{~nm}$, which will cause the area function to rise very sharply at low penetration depths, thus the initial hardness and Young's modulus will decrease sharply at the shallow indentation depth.

\subsection{Al film on different substrates}

The indentation depths on $\mathrm{Al}$-glass and $\mathrm{Al}-\mathrm{Si}$ are twice of the film thicknesses, depending on the thickness of the film. The load and stiffness recorded as a function of indentation depth during the experiments are used to calculate the hardness and Young's modulus of the films.

Fig. 2 shows the relation curves between the unloading contact stiffness, $S$, and the normalized indenter displacement, $h / t$, for the $244.7 \mathrm{~nm} \mathrm{Al} \mathrm{films} \mathrm{on} \mathrm{glass} \mathrm{and} \mathrm{silicon}$ substrates. As shown in Eq. (2), the unloading stiffness is directly proportional to the reduced modulus and the square root of the contact area. It is expected to increase linearly with indentation depth for the system, in which the film has a similar Young's modulus with that of the substrate. If the film and the substrate have different Young's modulus, the measured unloading contact stiffness will deviate from linearity as the indentation increases. As shown in Fig. 2, the unloading contact stiffness of Al film on silicon substrate deviates from linearity since the silicon substrate is stiffer than the $\mathrm{Al}$ film and that of Al-glass is almost linear with respect to indentation depth because $\mathrm{Al}$ and glass have similar Young's modulus.

The composite hardness of the film-substrate system should change when the film thickness changes. The hardnesses and Young's moduli for Al-glass systems with different film thicknesses are shown in Fig. 3. Due to the fluctuation of hardness for the thinnest Al film with thickness of $52.3 \mathrm{~nm}$, we give the composite hardnesses only for

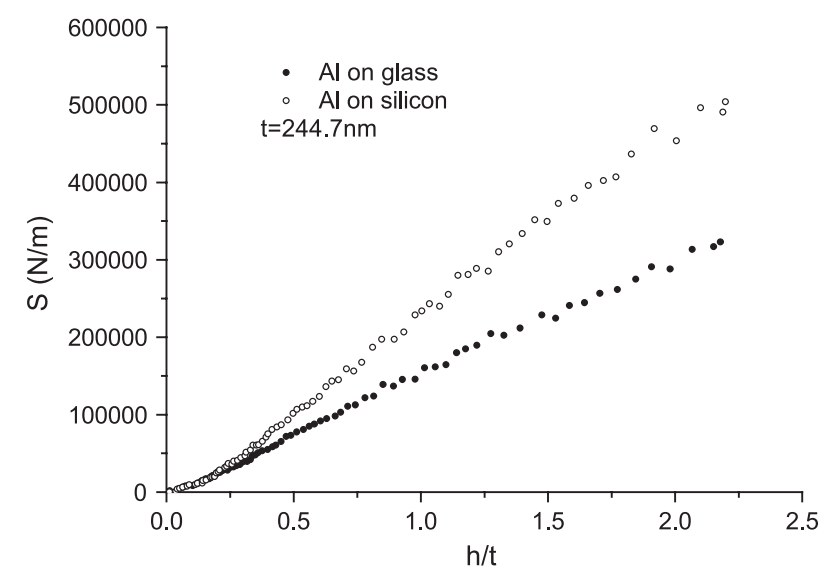

Fig. 2. The unloading stiffness versus normalized indentation depth for $\mathrm{Al}$ films on glass and silicon substrates with film thickness $244.7 \mathrm{~nm}$.

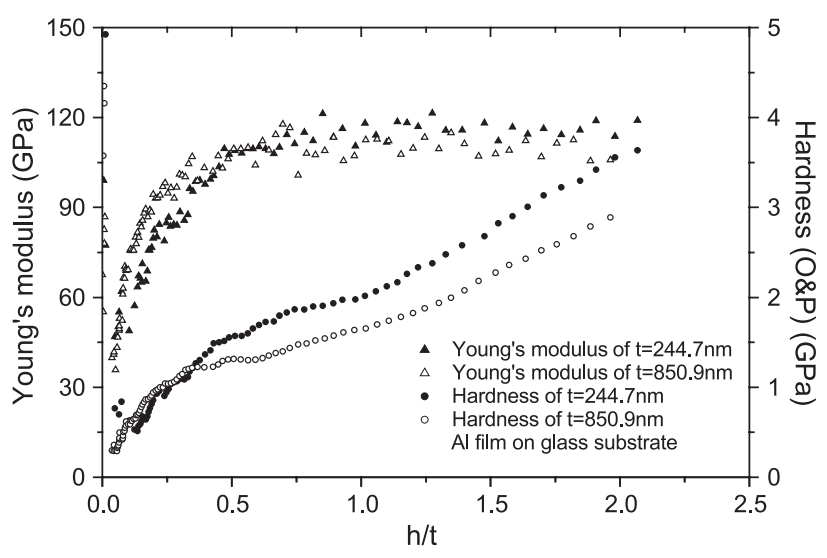

Fig. 3. Hardness and Young's modulus versus normalized indentation depth for Al films with thicknesses 244.7 and $850.9 \mathrm{~nm}$ on glass substrate.

the other two kinds of $\mathrm{Al}$-glass systems with film thicknesses of 244.7 and $850.9 \mathrm{~nm}$, as a function of the normalized indentation depth, $h / t$. From Fig. 3, one can see that the composite hardness decreases with increasing indentation depth at extremely small depths, which has been explained to be size effects in Refs. [14,22,42]. The hardness keeps a constant value only in a very small region, then begins to increase with the increasing indentation depth. This kind of increase in hardness is caused by the influence of the substrate. When the indentation depth is near the film thickness, a more significant increase in hardness is observed, which is due to the penetration into the hard substrates. One can see easily also that the hardness increases as the film thickness decreases, which can be explained by strain gradient effects or the Hall-Petch effects. In Fig. 3, Young's modulus for both thicknesses of Al films seems to be a constant except at the initial stage, which is due to the indenter tip round and the other experiment resolution. One can conclude that $\mathrm{Al}$ film has a similar Young's modulus with the glass substrate according to Eq. (3) since the curves keeps a horizontal line when $h / t>0.5$.

The composite hardnesses and Young's modulus of Alsilicon systems with different film thicknesses are shown in Fig. 4, where only the composite hardnesses for two kinds of Al-silicon systems with the film thicknesses of 244.7 and $850.9 \mathrm{~nm}$, as a function of the normalized indentation depth $h / t$ are shown due to the fluctuation of hardness for the thinnest Al film with thickness of $52.3 \mathrm{~nm}$. The phenomena about the composite hardness in Fig. 4 are the same as that found in Fig. 3, but the phenomena about the Young's modulus for Al-silicon system is different from that in Fig. 3. Since the Al film has a different Young's modulus from the Si substrate, the reduced Young's modulus increases with the increasing indentation depth, which is due to the substrate effects. The reduced Young's modulus is not influenced by the ratio of the indentation depth to the film thickness for $\mathrm{Al}-\mathrm{Si}$ systems.

For Al film of $244.7 \mathrm{~nm}$ thickness on glass and silicon substrates, the indentation loadings are the same in the 


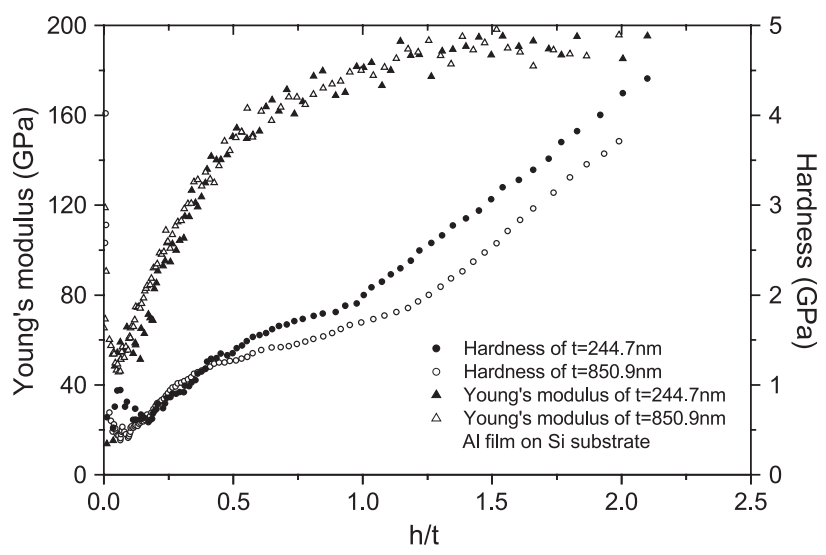

Fig. 4. Hardness and Young's modulus versus normalized indentation depth for Al films with thicknesses 244.7 and $850.9 \mathrm{~nm}$ on silicon substrate.

region of $h \leq t$ when the $\mathrm{Al}$ films have the same thicknesses though on different substrates. When $h>t$, i.e., the indenter tip penetrates into the substrates, indentation loading is larger on stiffer substrate than on softer substrate; therefore, one can see that the substrate effect works. In Fig. 5, plots of hardnesses and Young's modulus versus the normalized indentation depth are shown for the films of the same thickness but on different substrates. One can see that the hardnesses for the films of the same thickness and on different substrates are almost the same when the indentation depth $h \leq t$; when $h>t$, the hardness is larger on stiffer substrate than on softer substrate. However, the Young's moduli for the films of the identical thicknesses deviate from each other as long as $h \leq 0.25 t$, which means that the measured elastic moduli are more strongly affected by the substrate, as compared to the hardness.

Fig. 6 shows the parameter, $P / S^{2}$, as a function of normalized indentation depth, $h / t$, for the $244.7 \mathrm{~nm}$ Al films on glass and silicon substrates. After initial drop, the value of $P / S^{2}$ scales inversely with the modulus of the substrates, that is the value of $P / S^{2}$ is larger for $\mathrm{Al}$ film on glass substrate than that for $\mathrm{Al}$ film on silicon substrate. It is

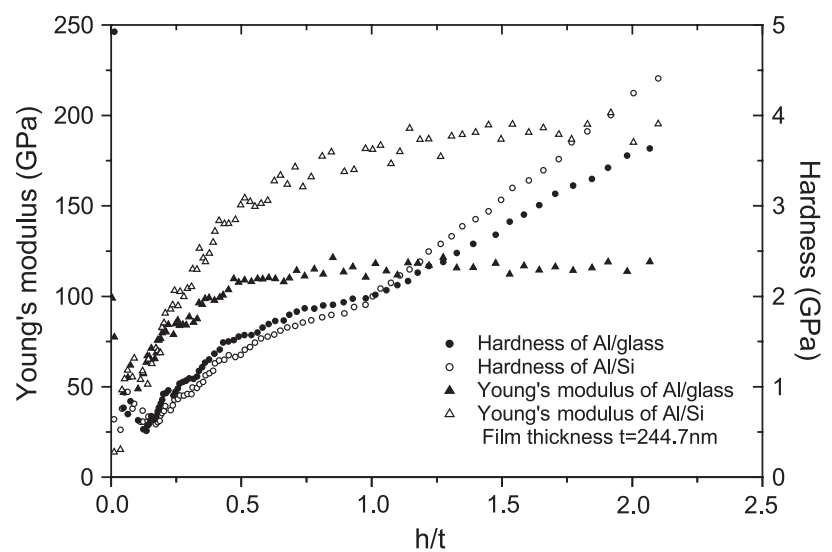

Fig. 5. Hardness and Young's modulus versus normalized indentation depth for Al film with thickness $244.7 \mathrm{~nm}$ on glass and silicon substrates.

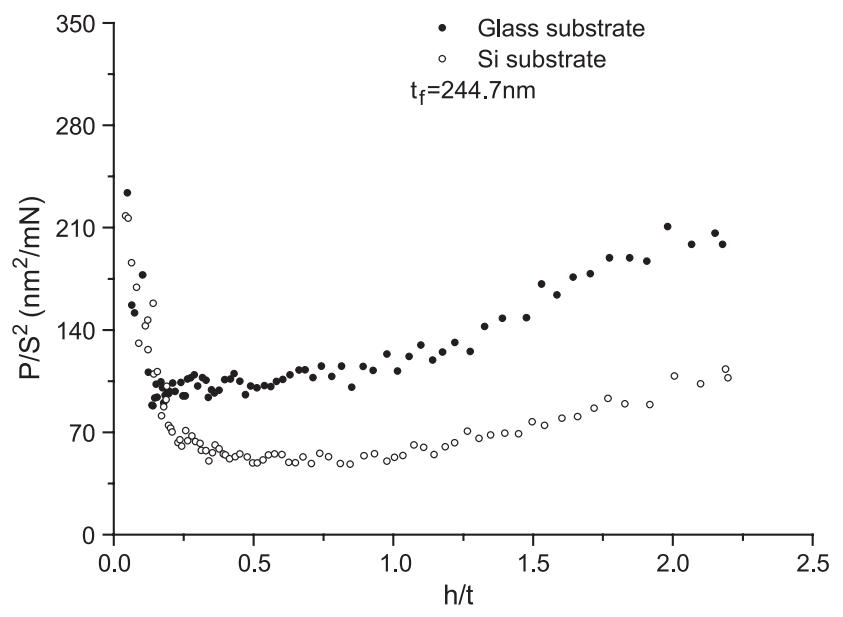

Fig. 6. $P / S^{2}$ versus normalized indentation depth for Al film with thickness $244.7 \mathrm{~nm}$ on glass and silicon substrates.

reasonable according to Eq. (7), that is, $P / S^{2}$ is inversely proportional to the square of the reduced modulus, $E_{\mathrm{r}}^{2}$.

\subsection{W film on different substrates}

In the present study, two kinds of W films of thicknesses 66.5 and $280 \mathrm{~nm}$ on glass and silicon substrates are investigated, respectively. This kind of film-substrate system represents hard film and soft substrate, which is contrary to the $\mathrm{Al}$ film on glass or silicon substrate.

For the load-displacement response of $280 \mathrm{~nm} \mathrm{~W}$ films on glass and silicon substrates with the indentation displacement twice of the film thickness, even at small indentation depth, the substrate effects are significant, as is different from $\mathrm{Al}-$ glass and $\mathrm{Al}-\mathrm{Si}$, which means that yielding of the soft substrate occurs at small indentation depth, as is different from soft film on hard substrate.

Hardness and Young's modulus are shown in Fig. 7 for $280 \mathrm{~nm}$ W film on glass and silicon substrates. The results are plotted as a function of the normalized indentation depth. The hardness increases at a small depth. When the

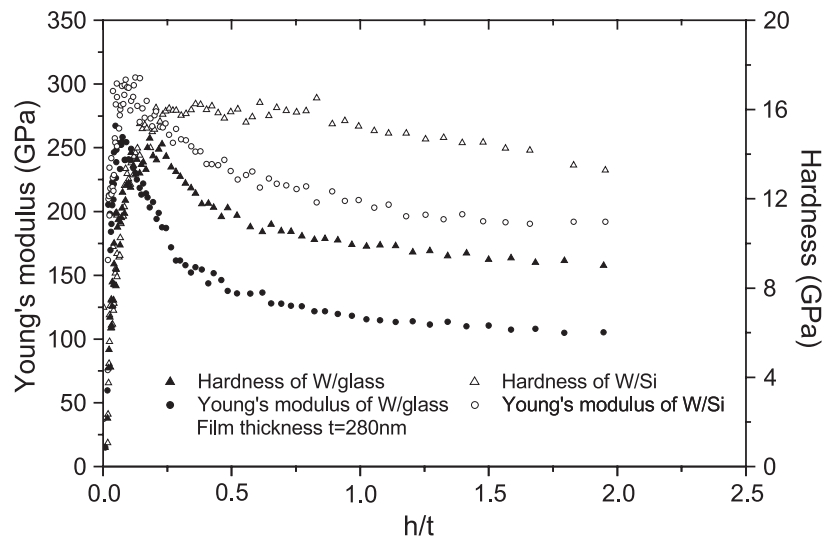

Fig. 7. Hardness and Young's modulus versus normalized indentation depth for W film with thickness $280 \mathrm{~nm}$ on glass and silicon substrates. 


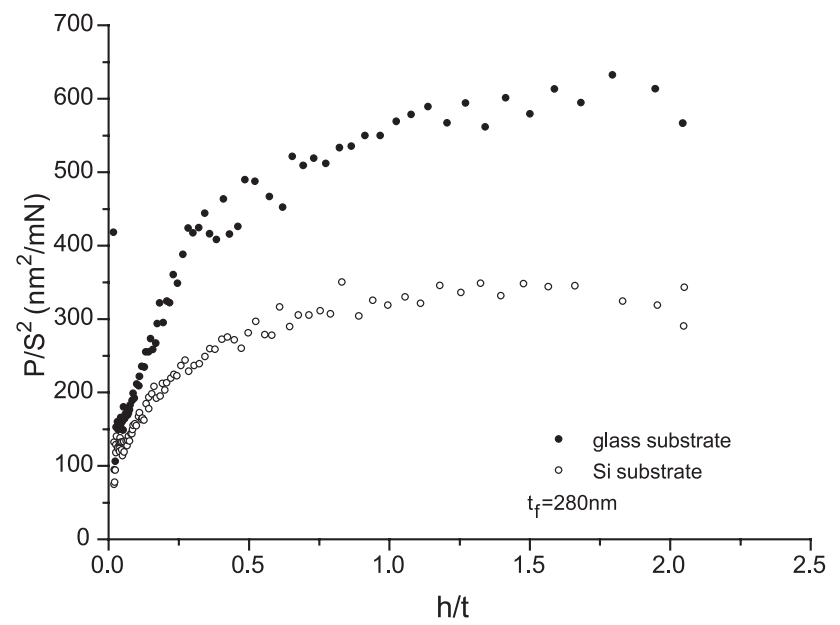

Fig. 8. $P / S^{2}$ versus normalized indentation depth for $\mathrm{W}$ film with thickness $280 \mathrm{~nm}$ on glass and silicon substrates.

hardness of W/glass system gets to $14 \mathrm{GPa}$ and $h / t=0.25$, it decreases as the indentation depth increases. For W/silicon system, after the hardness gets to about $16 \mathrm{GPa}$, where $h /$ $t=0.25$, it begins to decrease as the indentation depth increases. The observed behavior is expected considering the influence of the substrate hardness on the measured hardness since both glass and silicon substrates are softer than W material. The Young's modulus measured using the Oliver and Pharr method on glass and silicon substrates shows that even at a small indentation depth, the Young's modulus on different substrates do not match-up, which is different from what has been observed in the case of soft films on hard substrates. The effect of the substrate is observed over the entire range of the indentation depths.

Fig. 8 is a plot of the parameter, $P / S^{2}$, versus the normalized indentation depth for the $280 \mathrm{~nm} \mathrm{~W}$ film on glass and silicon substrates. One can see that the parameters $P / S^{2}$ for both film-substrate systems increase with the indentation depth. It is reasonable according to Eq. (7) since the parameter, $P / S^{2}$, is inversely proportional to the square of the reduced modulus. The influence of the square of the reduced modulus is larger than that of the composite hardness on $P / S^{2}$, which leads to the increasing value of $P / S^{2}$, though the composite hardness reduces as the indentation depth increases and $P / S^{2}$ is proportional to the composite hardness.

\subsection{Results using constant Young's modulus assumption}

As pointed by Ref. [14] that there is a problem in determining the intrinsic hardness of thin films, as the measurement is influenced by the properties of the substrates, especially when the film is very thin. The hardness measurement of a soft film is enhanced by the hard substrate while the hardness of a hard film is reduced by the soft substrate. True contact area cannot be obtained precisely during the experiment because all the measurements are based on the Oliver and Pharr method. The true contact depth is underestimated for a soft film on a hard substrate system and overestimated for a hard film on a soft substrate system comparing to the contact depth calculated using the Oliver-Pharr method. Thus, the hardness is overestimated for a soft film on a hard substrate and underestimated for a hard film on a soft substrate.

In order to avoid the calculation of the true contact area, the method proposed by Joslin and Oliver [9] is used in this section, i.e., using the parameter, $P / S^{2}$. This parameter can be used only when the material is elastically homogeneous and the Young's modulus of the indenter is known. In this study, we choose Al-glass system to check the results, since Al film has a similar Young's modulus with that of glass.

We take the Young's modulus of Al and glass as $E_{\mathrm{Al}}=E_{\text {glass }}=88.5 \mathrm{GPa}$, which is the measured data of single glass substrate, though the Young's modulus of $\mathrm{Al}$ in the literature is around $75 \mathrm{GPa}$. Poisson's ratio is approximately taken as $v_{\mathrm{Al}}=v_{\text {glass }}=0.3$. The Young's modulus of the Berkovich indenter is taken as $1140 \mathrm{GPa}$ and the Poisson's ratio is 0.07 , which is taken from the literature. The constant $\beta$ is taken as 1.034 , which is corresponding to the Berkovich indenter geometry. According to Eq. (6), the hardness is determined from the load, $P$, and the unloading contact stiffness, $S$, through the parameter, $P / S^{2}$. Fig. 9 shows the hardness as a function of the normalized indentation depth for the two different $\mathrm{Al}$ film thicknesses, which is calculated by means of the constant Young's modulus assumption. From Fig. 9, one can see that at small indentation depth, the composite hardness decreases as the indentation depth increases, which is due to the size effects or the experiment resolution. At a deeper indentation, the hardnesses get to constant values of about $1.1 \mathrm{GPa}$ for the $244.7 \mathrm{~nm}$ film and $0.9 \mathrm{GPa}$ for the $850.9 \mathrm{~nm}$ film. The hardnesses are approximately constant until the indentation depth is about 0.75 times the film thickness. Then the hardness starts to increase with increasing indentation depth. The plateau and subsequent increases are explained by Refs. $[14,42]$ to be caused by hardening associated with the strong gradients of plastic

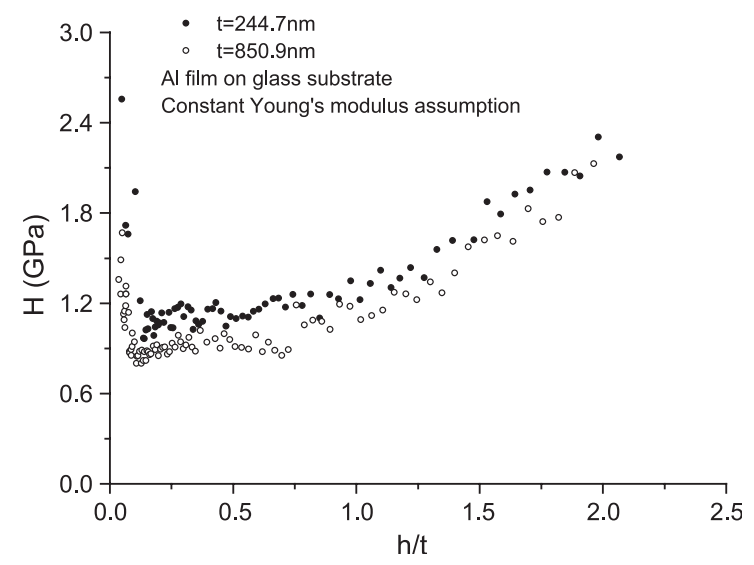

Fig. 9. Hardness calculated using the constant Young's modulus assumption versus the normalized indentation depth for Al films with thicknesses 244.7 and $850.9 \mathrm{~nm}$ on glass substrate. 
strain in film between the indenter and the substrate. When the indentation depth gets to the film-substrate interface, more significant increase in hardness with the increasing indentation depth can be observed, which is mainly due to the indenter penetration into the harder substrate. From the analysis, we could take the plateau values to be the true mechanical properties of the film since the other stages belong to transitional regions including the film and substrate characteristics.

\section{Conclusions}

In our attempts to understand the mechanical properties of film-substrate systems, we have chosen two kinds of films with different film thicknesses on two kinds of substrates, respectively. They include soft film on hard substrate and hard film on soft substrate. Nanoindentation experiment method is used. By depositing the same film with the same thickness on different substrates, we can show how the substrate properties influence the measured film properties.

It is found that for a soft film on a hard substrate the hardness decreases at a small indentation depth, then keeps a constant value, after which the hardness increases with increasing indentation depth, especially when the indentation depth is equal to or larger than the film thickness. As for the two kinds of systems, that is, the same film with same thickness on different substrates, the hardness is almost the same for the two kinds of systems before the indenter penetrates into the substrate but the Young's modulus begins to be affected in a small indentation depth, which means that the plastic deformation occurs in the film when $h \leq t$ and the substrate has little effects on the measured hardness.

For the hard film on the soft substrate, the hardness increases at a small indentation depth; after it reaches a maximum value, it decreases with increasing indentation depth. The hardness decreases more quickly when the substrate is softer. For the two kinds of systems, that is, the same film with same thickness on different substrates, both the composite hardness and the Young's modulus are affected by the substrates, which also means that the substrate yields before the film.

For the same film but of different thicknesses on the same substrate, we can show how the film thickness affects the measured composite hardness and Young's modulus. It is found that the hardness of the film increases when the film thickness decreases but the Young's modulus is not influenced by the film thickness.

Hardness is observed to be constant only in a very small region and the indentation depth less than the film thickness, which is due to the fact that the contact areas are determined by the Oliver and Pharr method. The Joslin and Oliver method is used in the present paper to avoid the calculation of the contact area and the constant Young's modulus assumption is used; one can easily determine the hardness from a measure of both the load, $P$, and contact stiffness, $S$. Using Eq. (6), we find that the hardness for $\mathrm{Al}$-glass system keeps a constant value in the region $h / t=0.1-0.75$.

\section{Acknowledgements}

The authors would like to express their gratitude to $\mathrm{Y}$. Yu and T.H. Zhang for their help in doing the experiments and to the National Science Foundation of China (No. 10202023; No. 10272103), Key Project from the Chinese Academy of Sciences (Grant No. KJCX2-SW-L2), Excellent Post-doctoral Research-Starting Fund of CAS for financial supports. W. Wang, who works in Microelectronics Processing Laboratory of the Institute of Microelectronics, Peking University, is acknowledged for her help in depositing the films. Thanks are also due to the unknown reviewers for their helpful comments.

\section{References}

[1] J.B. Pethica, R. Hutchings, W.C. Oliver, Philos. Mag., A 48 (1983) 593.

[2] M.F. Doerner, W.D. Nix, J. Mater. Res. 1 (1986) 601

[3] M.F. Doerner, D.S. Gardner, W.D. Nix, J. Mater. Res. 1 (1986) 845.

[4] W.C. Oliver, G.M. Pharr, J. Mater. Res. 7 (1992) 1564.

[5] T.Y. Tsui, W.C. Oliver, G.M. Pharr, Mater. Res. Soc. Symp. Proc. 436 (1997) 147.

[6] P.J. Burnett, D.S. Rickerby, Thin Solid Films 148 (1987) 51.

[7] J.L. Hay, Me O'Hern, W.C. Oliver, Mater. Res. Symp. Proc. 522 (1998) 27.

[8] J.C. Hay, G.M. Pharr, Mater. Res. Symp. Proc. 505 (1998) 65.

[9] D.L. Joslin, W.C. Oliver, J. Mater. Res. 5 (1990) 123.

[10] A.M. Korsunsky, M.R. McGurk, S.J. Bull, T.F. Page, Surf. Coat. Technol. 99 (1998) 171

[11] J. Mencik, D. Munz, E. Quandt, E.R. Weppelmann, M.V. Swain, J. Mater. Res. 12 (1997) 2475.

[12] T.F. Page, et al., Mater. Res. Soc. Symp. Proc. 522 (1998) 53.

[13] T.Y. Tsui, G.M. Pharr, J. Mater. Res. 14 (1999) 292.

[14] R. Saha, W.D. Nix, Acta Mater. 50 (2002) 23.

[15] A. Nolshakov, G.M. Pharr, J. Mater. Res. 13 (1998) 1049

[16] P.J. Burnett, D.S. Rickerby, Thin Solid Films 148 (1987) 41.

[17] H. Gao, C. Cheng-Hsin, L. Jin, Int. J. Solids Struct. 29 (1992) 2471.

[18] R.B. King, Int. J. Solids Struct. 23 (1987) 1657.

[19] K.C. Tang, R.D. Arnell, Thin Solid Films 356 (1999) 263.

[20] Y.T. Cheng, C.M. Cheng, Appl. Phys. Lett. 356 (1998) 263.

[21] H.Y. Hu, S.C. Sanday, B.B. Rath, J. Mech. Phys. Solids 38 (1990) 745 .

[22] R. Saha, Z.Y. Xue, Y. Huang, W.D. Nix, J. Mech. Phys. Solids 49 (2001) 1997

[23] W.D. Nix, Metall. Trans. 20A (1989) 2217.

[24] J.A. Schweitz, MRS Bull. 17 (1992) 33.

[25] G.M. Pharr, W.C. Oliver, MRS Bull. 17 (1992) 28.

[26] J. Mencik, Mech. Comp. With Treated or Coated Surfaces, Kluwer Academic Publishing, Dordrecht, 1996.

[27] J.S. Field, M.V. Swain, J. Mater. Res. 8 (1993) 297

[28] J. Mencik, M.V. Swain, Mater. Forum 18 (1994) 277.

[29] M.V. Swain, E. Weppelmann, Mater. Res. Soc. Symp. Proc. 308 (1993) 177

[30] D. Lebouvier, P. Gilormini, E. Felder, Thin Solid Films 172 (1983) 227. 
[31] A.K. Bhattacharya, W.D. Nix, Int. J. Solids Struct. 24 (1988) 881.

[32] T.A. Laursen, J.C. Simo, J. Mater. Res. 7 (1992) 618.

[33] B. Jonsson, S. Hogmark, Thin Solid Films 114 (1984) 257.

[34] P.M. Sargent, in: P. Blau, B.R. Lawn (Eds.), Microindentation Techniques in Material Science and Engineering, American Society for Testing and Materials, Philadelphia, PA, 1986, p. 160.

[35] P.J. Burnett, T.F. Page, J. Mater. Sci. 19 (1984) 845.

[36] B.R. Lawn, A.G. Evans, D.B. Marshall, J. Am. Ceram. Soc. 63 (1980) 198.
[37] B.D. Fabes, W.C. Oliver, R.A. McKee, F.J. Walker, J. Mater. Res. 7 (1992) 3056

[38] N.G. Chechenin, J. Bottiger, J.P. Krog, Thin Solid Films 261 (1995) 227.

[39] I.N. Sneddon, Int. J. Eng. Sci. 3 (1965) 47

[40] R. Venkatraman, J.C. Bravman, J. Mater. Res. 7 (1992) 2040.

[41] S.D. Mesarovic, N.A. Fleck, Proc. R. Soc. Lond. 455 (1999) 2707.

[42] S.H. Chen, L. Liu, T.C. Wang, Acta Mater. 52 (5) (2004) 1089. 